\title{
On the present and future of dissolution-DNP
}

\section{Ardenkjær-Larsen, Jan Henrik}

\section{Published in:}

Journal of Magnetic Resonance

Link to article, DOI:

10.1016/j.jmr.2016.01.015

Publication date:

2016

Document Version

Peer reviewed version

Link back to DTU Orbit

Citation (APA):

Ardenkjær-Larsen, J. H. (2016). On the present and future of dissolution-DNP. Journal of Magnetic Resonance, 264, 3-12. https://doi.org/10.1016/j.jmr.2016.01.015

\section{General rights}

Copyright and moral rights for the publications made accessible in the public portal are retained by the authors and/or other copyright owners and it is a condition of accessing publications that users recognise and abide by the legal requirements associated with these rights.

- Users may download and print one copy of any publication from the public portal for the purpose of private study or research.

- You may not further distribute the material or use it for any profit-making activity or commercial gain

- You may freely distribute the URL identifying the publication in the public portal

If you believe that this document breaches copyright please contact us providing details, and we will remove access to the work immediately and investigate your claim. 


\title{
On the present and future of dissolution-DNP
}

\author{
Jan Henrik Ardenkjaer-Larsen \\ Technical University of Denmark, Department of Electrical Engineering, Kgs Lyngby, \\ Denmark and GE Healthcare, Brøndby, Denmark
}

Corresponding address:

Technical University of Denmark

Ørsted Plads, bldg. 349, room 126

DK-2800 Kgs Lyngby

Denmark

Keywords: Dissolution-DNP, hyperpolarization, polarizer 
[Type here]

\section{Abstract}

Dissolution-DNP is a method to create solutions of molecules with nuclear spin polarization close to unity. The many orders of magnitude signal enhancement have enabled many new applications, in particular in vivo MR metabolic imaging. The method relies on solid state Dynamic Nuclear Polarization at low temperature followed by a dissolution to produce the room temperature solution of highly polarized spins. This work describes the present and future of dissolution-DNP in the mind of the author. The article describes some of the current trends in the field as well as outlines some of the areas where new ideas will make an impact. Most certainly, the future will take unpredicted directions, but hopefully the thoughts presented here will stimulate new ideas that can further advance the field. 
[Type here]

\section{Introduction}

Since the earliest days of magnetic resonance, various concepts of enhancing the signal-to-noise ratio by increasing the nuclear spin polarization above thermal equilibrium have been conceived and investigated. Magnetic Resonance is inherently an insensitive method. The energy of a nuclear magnetic moment in any magnetic field that can be produced, is small compared to thermal energy at room temperature, causing the nuclear alignment to be weak. For spin $1 / 2$ the spin polarization is expressed by the MaxwellBoltzmann statistics

$$
P=\tanh \left(\frac{\gamma \hbar B_{0}}{2 k_{B} T}\right)
$$

The detected signal is proportional to the polarization, $P$. At a typical clinical imaging field strength of $3 \mathrm{~T}$ and room temperature the polarization of protons is $10 \mathrm{ppm}$ and for carbon13 it is $2.5 \mathrm{ppm}$. At a typical NMR magnetic field strength of e.g. $14.1 \mathrm{~T}\left(600 \mathrm{MHz}{ }^{1} \mathrm{H}\right)$ the proton polarization is $47 \mathrm{ppm}$ and the carbon polarization is $12 \mathrm{ppm}$. The potential for sensitivity improvement is therefore tremendous; three to five orders of magnitude depending on the nucleus and field strength.

Trivially, the polarization can be increased by raising the magnetic field and lowering the temperature, but there is a limited playground in conventional NMR and MRI. Currently, the highest NMR magnetic field strength is $23 \mathrm{~T}$ and for whole body MRI it is $9.4 \mathrm{~T}$. Only in solid state NMR is there any significant room to increase the polarization by lowering the temperature $[1,2]$.

Hyperpolarization signifies a spin polarization that deviates strongly from thermal equilibrium. A characteristic of hyperpolarization is that the polarization is often created ex situ, and therefore that cannot be recreated to the initial state. It is a game of "use it 
[Type here]

(smart), or lose it". There are situations where the sample (or hyperpolarization) can be recycled, e.g. melting-DNP [3-5] or SABRE [6], but in most cases, the hyperpolarization process is irreversible or destructive: e.g. in vivo metabolism, chemical or cellular reactions, dissolution and hydrogenation.

Several hyperpolarization methods have been developed. Brute force polarization has been investigated to reach high nuclear polarization by going to milliKelvin temperatures in strong magnetic fields. Other schemes like optical pumping, dynamic nuclear polarization (DNP) and parahydrogen-induced-polarization (PHIP) are also wellknown to most scientists in the field. Some of these methods have a long history and have had practical applications like the proton and helium magnetometers and polarized targets in nuclear physics studies.

Hyperpolarization has already made an impact in the fields of chemistry, biology and medicine. The methods have enabled experiments that could not be done otherwise, and have e.g. given new insight into the biochemistry of normal and abnormal cell metabolism. There is all reason to believe that this field of research will continue for many years with important discoveries yet to come.

\section{Hyperpolarization}

Hyperpolarization entered the field of in vivo Magnetic Resonance Imaging with optical pumping of the noble gases $\left({ }^{3} \mathrm{He}\right.$ and $\left.{ }^{129} \mathrm{Xe}\right)$ for lung imaging [7]. Optical pumping is a scheme of transferring angular momentum from photons (narrow line laser) to electrons in a vapor of e.g. an alkali metal. The electron spin of the alkali metal can be fully polarized and will polarize the nuclear spin of the noble gas by collisional spin exchange. The nuclear signal is thereby enhanced by many orders of magnitude over thermal equilibrium at room 
[Type here]

temperature. Kastler was awarded the Nobel Prize in 1953 for this work. The instrumentation for optical pumping will not be described here, but it is worth noting that in this community, freezing out the hyperpolarized gas for accumulation and subsequently sublimating of the solid with preservation of the polarization is common practice.

PHIP is another elegant method of creating highly polarized nuclear spins in solution through the spin order of para-hydrogen [8,9]. At a temperature of ca. $20 \mathrm{~K}$ the $S=0$ ground state (para-hydrogen) of molecular hydrogen is fully populated. The conversion from orthohydrogen $(S=1)$ to para-hydrogen is slow and a catalyst is needed (e.g. iron oxide). This also means that para-hydrogen is easily stored and transported at ambient temperature and magnetic field. The spin order of para-hydrogen can then be transferred to a third (or more) spin by either a chemical hydrogenation of an unsaturated bond or via a transient magnetic interaction mediated by a catalyst [6]. The instrumentation of PHIP is simple, essentially only requiring para-hydrogen to be mixed with the substrate and a catalyst. The introduction of PHIP into the field of medical imaging happened parallel to dissolution-DNP [10].

Brute-force $[11,12]$ polarization has been proposed as an alternative to dissolutionDNP. In this scheme, the polarization is achieved by cooling the sample to millikelvin temperatures in a high magnetic field. The challenge is to overcome the excessively long $T_{1}$ at low temperature (days) to reach thermal equilibrium. In principle, brute-force polarization does not require formulation with a polarizing agent, like in DNP, but in practice, a relaxation enhancing agent is required to shorten $T_{1}$. Nor does the magnetic field need to be homogenous.

\section{Dissolution-DNP}


[Type here]

Our work on hyperpolarization evolved out of a different context. The group of Prof Golman in Malmö, Sweden, started in the mid-eighties, along with several other groups, to develop Overhauser enhanced MRI $[13,14]$. The idea was to exploit the Overhauser effect to enhance the water proton signal in low field MRI by injecting a free radical contrast agent. In the case of an electron spin and the proton, the enhancement can be up to -329 when the coupling is entirely dipolar. A whole body field-cycling magnet that can generate a static magnetic field of e.g. $15 \mathrm{mT}$, does not permit ${ }^{1} \mathrm{H}$ images of useful quality in a reasonable time. When a radical is injected a hundred-fold Overhauser enhancement is obtained when saturating the paramagnetic resonance (e.g. at $7 \mathrm{mT}$ and $200 \mathrm{MHz}$ ) and in vivo high quality images of tissue and vasculature can now be obtained in a few minutes. In Overhauser MRI the main challenge is to develop paramagnetic molecules with a single narrow EPR line and high relaxivity. Derivatives of the trityl meet all requirements of an Overhauser contrast agent: high chemical stability in a biological system, low toxicity and a single narrow EPR line. It is a large molecule with molecular weight of $\sim 1500 \mathrm{~g} / \mathrm{mol}$ and a radius of about $5 \AA$. Hyperfine couplings are eliminated by removing all protons close to the radical center. The main reason this project was stopped was a too low relaxivity requiring too large a dose of the radical (despite its low toxicity). Overhauser MRI is limited by the absorption of RF energy restricting the magnetic field to less than ca. $10 \mathrm{mT}(280 \mathrm{MHz})$. However, the largest magnetization is obtained close to the dispersion point of the coupling factor, which is closer to one Tesla depending on the dynamics of the system. This led to our first approach to hyperpolarization; ex vivo Overhauser enhancement of water as a contrast agent for angiography and perfusion. However, imaging of biological molecules at physiological concentrations would allow MRI to enter the field of molecular imaging, and add something unique. The only issue was that the limited Overhauser enhancement would not deliver the 
[Type here]

required improvement in sensitivity. The vision of hyperpolarized Metabolic MR was conceived before a method of hyperpolarizing nuclear spins in solution was available.

The idea for dissolution-DNP came in the fall of 1997 by sudden inspiration. The book of Abragam and the early seventies polarized target literature on impressive solidstate polarizations in organic materials at low temperature [15] was animating, and led to the idea that the temperature jump and solid to solute transition could be fast enough to preserve the polarization. This was a completely new field of research for us, and before any experiments could be done, we had to build the dissolution-DNP polarizer. Injecting superheated solvent into the cryostat was not an obvious thing to do, but gradually understanding the criticality of insulating the sample and isolating regions as well as the time scale of the experiment gave us the confidence that this could work. Dissolution-DNP is based on the notion that a $1 \mathrm{~K}$ "ice cube" can be dissolved in a fraction of a second or short on $T_{1}$ time scale. Secondly, that injection of superheated solvent into a cryostat is harmless if properly carried out. A rough calculation of the thermodynamics gives an impression of the scale of the problem: The latent and sensible heat of a typical organic material when going from $1 \mathrm{~K}$ to RT is about $1600 \mathrm{~J} / \mathrm{g}$. If this heat is dumped into the helium bath, several hundred $\mathrm{mL}$ of liquid helium is evaporated. However, in the dissolution of e.g. $1 \mathrm{~g}$ of propanediol it becomes clear that the $1600 \mathrm{~J}$ is a small amount of energy to supply, when considering the $4.2 \mathrm{~J} / \mathrm{g} / \mathrm{K}$ specific heat capacity of water. $1 \mathrm{~g}$ of propanediol dissolved in 50 $\mathrm{mL}$ of water only reduced the solvent temperature by $8 \mathrm{~K}$. This shows that excess energy is available and dissolution efficiency relies on other factors.

The dissolution-DNP method was published in 2003 [16]: “Increase in signal-to-noise ratio of $>10,000$ times in liquid state NMR"; a title that attracts attention, but could be misleading. Nevertheless, the message was that a new method of polarizing spins in solution 
[Type here]

had been devised that could enhance the nuclear spin polarization to tens of percent; or 10,000-fold over thermal equilibrium at any achievable magnetic field strength. The example was natural abundance urea that was polarized to $20 \%$ : a 24,000 -fold enhancement over $9.4 \mathrm{~T}$. The thermal spectrum on the same sample took 65 hours to acquire with a thousand-fold lower SNR. 27 years of averaging would have been required to reach the same SNR. This translates into a huge increase in sensitivity.

\section{How do we reach unity polarization fast?}

The dissolution-DNP method implies polarizing the nuclear spins in the solid state by DNP at low temperature, e.g. 1-2 K, in a high magnetic field, 3-7 T. At temperature and field conditions that are easily achievable, the electron spin polarization is almost unity. Microwave irradiation close to the paramagnetic resonance causes nuclear spin populations to redistribute and approach the electron spin polarization. This is a slow process at low temperature, minutes to hours, but eventually when high solid state polarization has been achieved, the sample can be dissolved. This is a fast process, a few seconds, on the time scale of nuclear relaxation, and the polarization is preserved into the liquid state. However, the strong nuclear spin polarization in the liquid state relaxes back to thermal equilibrium. The instrumentation is shown in fig $1 \mathrm{~A}$.

Choosing the optimal temperature and magnetic field conditions for DNP is not evident. However, recent research suggests that a higher magnetic field strength is beneficial and more easily achieved than a lower temperature. This has been demonstrated for both trityls $[17,18]$ and nitroxides $[19,20]$. The nuclear polarization increases quadratic with field even to a polarization above $70 \%$ for trityl (fig 2). The reason for the strong field dependence is probably the increase of the nuclear $T_{1}$ at higher field, whereas the electron 
[Type here]

$T_{1}$ does not change significantly with magnetic field [21]. This is to be expected for the electron spin, where $T_{1}$ relaxation is by the direct effect. The nuclear $T_{1}$ is determined by the electron spin and therefore should exhibit a $\mathrm{B}_{0}^{2}$ dependence. The temperature dependence of DNP seems to follow $1 / T$ [18], which is consistent with both the nuclear and electron $T_{1}$ becoming proportionally longer with lower temperature. In this case, it is only the increased nuclear Boltzmann polarization that we benefit from, when lowering the temperature. The data suggests that it is the ratio of nuclear and electron $T_{1}$ that is limiting the efficiency of the DNP enhancement. It is likely that we will see in the coming years that dissolution-DNP is moving to even higher magnetic field strength. This is also supported by the availability of robust and compact microwave sources (solid state devices) with reasonable output power, as well as lower loss transmission (e.g. corrugated waveguides and/or low loss electromagnetic modes) and resonant structures. Dissolution-DNP needs to accommodate the dissolution components and isolate the hot solvent from the cryogenic environment. In addition, in particular in the case of the samples for biological studies, the sample is large compared to the wavelength of the microwave field ( $3.2 \mathrm{~mm}$ at $94 \mathrm{GHz}$ in free space). Therefore, resonant structures are difficult to implement, and so far, the microwave energy has been confined by oversized, low Q structures. It is likely, that dissolution-DNP will also see a development towards sample optimized resonant structures with high $Q$ that will allow the creation of strong microwave $B_{1}$ fields in the sample.

Narrow line radicals, like trityl and BDPA, are superior for direct polarization of low gamma nuclei in terms of the maximum polarization achievable. In some samples, it is advantageous to polarize the protons with nitroxides and cross-polarize to other nuclei. Cross-polarization (CP) has been demonstrated by several groups $[20,22-24]$ and high polarization in a few minutes can be achieved for these samples. In fig 3 it is shown how the 
[Type here]

${ }^{13} \mathrm{C}$ polarization is boosted by multiple $\mathrm{CP}$ contacts between ${ }^{1} \mathrm{H}$ and ${ }^{13} \mathrm{C}$. The challenge with $\mathrm{CP}$ has been the need for strong $\mathrm{B}_{1}$ fields at the NMR frequencies, required for efficient polarization transfer. The helium environment limits the power that can be applied due to ionization (probe arching). This scheme does provide a general path for high and fast nuclear polarization of low gamma nuclei through the protons. Further development of probe power handling, efficient transfer schemes and sample properties (e.g. ${ }^{1} \mathrm{H}$ density) will broaden the applicability and availability of the scheme.

More advanced schemes like the Integrated Solid Effect (ISE) [25] and Nuclear Orientation by Electron spin Locking (NOVEL) [26] are very demanding in terms of the microwave field. Therefore, these schemes have only been demonstrated at lower frequencies $(0.35 \mathrm{~T} / 9.3 \mathrm{GHz})$ where high $\mathrm{Q}$ cavities are available. Generally, these transfer schemes will be difficult to implement for the sample sizes of interest for most dissolutionDNP studies.

In combination with trityl, a low concentration of $\mathrm{Gd}(\mathrm{III})$, about a tenth of the concentration of the radical, has been shown to give a dramatic effect on the DNP enhancement: $50-100 \%$ increase in polarization $[27,28]$. Gd does not affect the nuclear $\mathrm{T}_{1}$, which is unchanged, and does not provide a DNP by itself. The EPR line of the trityl was already effectively homogeneous (fast spectral diffusion). However, the electron spin $T_{1}$ of the radical was dramatically shortened by Gd from about $1 \mathrm{~s}$ to $0.1 \mathrm{~s}$. The data suggests that the ratio of nuclear to electron $T_{1}$ is a critical parameter $[28,29]$. The addition of Gd or another paramagnetic transition metal to enhance the solid state DNP efficiency, is to eliminate or minimize the relaxation effect in the liquid state post dissolution. If the $\mathrm{Gd}$ is strongly chelated, the relaxivity to ${ }^{13} \mathrm{C}$ is low, and the typical quantities needed in the solid 
[Type here]

state has insignificant effect. However, caution needs to be taken to ensure stability of the chelate.

\section{Dissolution-DNP for chemistry, cell and small animal research}

Oxford Instruments built the first commercial dissolution-DNP polarizer, the Hypersense (fig 1). The design is similar to the original PNAS design (fig 1), but eliminates the operator variability in the dissolution by having pneumatically actuated docking of the dissolution stick and release of the heated solvent. This product has helped many groups to quickly access the technology and explore basic research and applications, primarily in the field of in vivo imaging. Other groups have built similar polarizers [30-33], but not deviated significantly in design principles. The Hypersense can be upgraded in various ways, e.g. with larger pumps to lower the base temperature from the specified $1.4 \mathrm{~K}$ to below $1.2 \mathrm{~K}$, or the dissolution system to allow larger samples [34]. However, the Hypersense operates at 3.35 T (94 GHz DNP), which is a field strength that at $1.4 \mathrm{~K}$ only provides $10-20 \%{ }^{13} \mathrm{C}$ polarization for typical samples doped with trityl. As previously mentioned the field has advanced to higher field strength. Another consideration with the original design is the leaching of paramagnetic ions from stainless steel parts in the dissolution system. The solvent vessel is heated to typically $180^{\circ} \mathrm{C}$ (10 bar vapor pressure for water) with a strong base. Small amounts of paramagnetic ions can typically be tolerated by adding e.g. EDTA to chelate these ions.

For chemistry applications, ultrafast transfer is essential to capture short $\mathrm{T}_{1}$ nuclei. With shielded magnets, the polarizer and NMR magnet can be placed within a few meters, in principle with the cryostats touching. The short distance minimizes the transfer time. An HPLC loop, fig 4, allows the concentrated front volume to be captured and shuttled to the 
[Type here]

NMR tube with minimal settling time. Transfer and stabilization within a few seconds has been demonstrated $[35,36]$, but sub-second performance should be feasible and will allow more signal to be retained, and fast relaxing nuclei to be captured. In [36] it was shown that the dissolution is highly inhomogeneous. For samples on the order of $10 \mu \mathrm{L}$ the mass recovery was $>50 \%$ in the first $440 \mu \mathrm{L}$ (optimized for $5 \mathrm{~mm}$ NMR tube), and that this volume could be isolated in the HPLC loop and transferred to the NMR tube. For cells, tissue and perfused organ applications it is equally important that the transfer and mixing is controlled efficiently to deliver reproducible substrate concentration and polarization [37]. The NMR bioreactor should be compatible with a $5 \mathrm{~mm}$ NMR probe and good shimming. For all of these in vitro applications, the combination with cryoprobes for further sensitivity gains make perfect sense.

In the case of small animal research, the quality of the in vivo results also depends on reproducible and fast transfer of the hyperpolarized agent. An automated transfer and injection system has been demonstrated [38]. This setup allows transfer of the hyperpolarized agent from the polarizer to the imager in a few seconds. The system also performs an NMR polarization measurement to correct for any variation in the polarization process and transfer. In particular, for agents with short $T_{1}$, like glucose and bicarbonate, the fast transfer is essential to obtain high quality in vivo spectra. The particular set up is demonstrated with ascorbic acid radical quenching [39], which has its limitations and disadvantages. Ascorbic acid may not be chemically compatible with the sample and the concentration of ascorbic acid needs to be hundreds of $\mathrm{mM}$ to effectively quench the nitroxide. The trityl is stable to ascorbic acid and is most efficiently removed by filtration or extraction. Transfer and infusion systems combined with means of radical removal will be important to the field. 
[Type here]

Typically, low field relaxation is not an issue (dipolar relaxation for small molecules in non-viscous media) and a magnetic field of a few Gauss between the polarizer and NMR is sufficient to prevent non-adiabatic transfer. For some spin (e.g. coupled to a quadrupolar nucleus like ${ }^{14} \mathrm{~N}$ or ${ }^{35} \mathrm{Cl} /{ }^{37} \mathrm{Cl}$ or with radicals with slow dynamics present), the low field relaxation time can be much reduced. In this case, the magnetic field between the polarizer needs to be elevated with e.g. permanent magnets [40]. Enriching with ${ }^{15} \mathrm{~N}$ will also remove the low field relaxation contribution (scalar relaxation of the second kind) [41], as well as prolong $T_{2}$ for improved signal acquisition [42]. In rare cases, e.g. long-lived-spin-states [43], a certain magnetic field may even be optimal for storing the sample, and the sample can be shuttled in and out of the magnet center to a location with the desired magnetic field strength for storage (and evolution of signal, chemical reactions, etc).

Dissolution with immiscible solvents is an elegant way of minimizing the dissolution volume and optimizing it for the application (e.g. $5 \mathrm{~mm} \mathrm{NMR} \mathrm{tube,} \mathrm{Shigemi} \mathrm{tube} \mathrm{or} \mathrm{injection}$ volume in a rodent) [44]. It is also an elegant way of extracting the radical to eliminate relaxation [45]. A $5 \mathrm{~mm}$ NMR tube requires $400 \mu \mathrm{L}$. Shigemi tubes, NMR tubes with smaller diameter or flow probes require even less volume. Dissolving in a volume of a few hundred microliter is challenging from the point-of-view of contact with the cold surfaces of the polarizer. In the current polarizer designs (e.g. Hypersense) the minimal dissolution volume is 3-4 $\mathrm{mL}$. To reduce the effective solute volume by an order of magnitude should be achievable using a two-phase system in the dissolution to extract the sample into the desired phase. The same principle can be applied for small animal studies, where expensive selectively labelled biomolecules need to be dissolved in e.g. $300 \mu \mathrm{L}$ buffer (maximum injection volume in a mouse). 
[Type here]

Labile radicals have been proposed as way of generating radicals in situ by means of light that are unstable at higher temperatures and therefore disappear in the dissolution or even in the solid at some elevated (above DNP conditions) temperature. In this way relaxation effects in the dissolution and liquid state are eliminated. It could also open up for relaxation switches in the solid state thereby enabling polarization and transport of the solid sample. In the case of pyruvic acid, and other keto-acids, UV generated radicals [46] are particularly interesting, fig 5. A high concentration of radicals is created with good properties for DNP. The radicals disintegrate at a temperature slightly above liquid nitrogen.

Recently, it has been proposed to use radicals (nitroxides) immobilized on the surface of silica particles [47]. These heterogeneous systems (HYPSO) allow easy separation of the radical, and also do not require any glassing solvent, fig 6 . This interesting development could likely be further optimized. However, the results suggest that the DNP efficiency is reduced. The build-up rate constant is reduced and the polarization level is lower than for the similar homogenous formulations. Likewise, it is possible that dissolution efficiency is affected since mobility and diffusion within the pore structure may reduce $T_{1}$.

It has also been demonstrated that the dissolution can take place external to the polarizer [48]. This was achieved in inhomogeneous systems like HYPSO or impregnated samples. Finely ground particles were impregnated with a nitroxide doped solvent, and the microcrystals were polarized by spin diffusion. The build-up rate constant is reduced, but a long ${ }^{13} \mathrm{C}$ relaxation of many hours is achieved, and the sample can pass through a low field region with a small loss of polarization when being retracted from the polarizer. This opens up the possibility of polarizing the solid sample in a centralized location and distributing samples to the point of use. For many applications the loss in polarization (during retraction 
[Type here]

and distribution) can be accepted, but for some, like in vivo imaging, the highest possible polarization is required.

Dual-center magnets have been developed to further reduce the transfer time. In this configuration the high field, high homogeneity NMR magnet is designed to have a lower, less homogeneous magnetic field at the edge of the primary magnet. It seems that this concept reduces flexibility with little advantage over two individual systems. However, the dual-center magnet may have advantages when combined with melting-DNP. Melting of the sample is feasible for small samples where heat conduction is sufficiently fast to melt the sample in a fraction of a second $[3,4]$. Instead of providing the heat through the solvent, heat is provided by conduction or absorption. The solid sample polarized by DNP, can be shuttled in the solid state from the low temperature DNP region, to the room temperature high field, high homogeneity NMR detection region where the sample is melted by e.g. a stream of warm gas. Laser heating has also been demonstrated. The hyperpolarized signal is acquired and the sample can be shuttled back to the DNP region for another cycle. This scheme allows averaging and multidimensional spectroscopy.

Melting-DNP makes perfect sense for mass-limited applications where ultimate sensitivity is the aim. Dissolution-DNP is advantageous in applications where the kinetics of chemical and biochemical reactions is the target.

\section{Is the technology viable in a clinical environment?}

For dissolution-DNP to become routine clinical practice, new polarizer technologies are required. A viable polarizer technology requires that handling of liquid cryogens is eliminated, that the process is automated and has minimal operator intervention, that the sample throughput is compatible with patient workflow and that quality control and sterility 
[Type here]

is ensured. Closed-cycle cryogenics is demanding considering a temperature of ca. $1 \mathrm{~K}$ and the high cooling power required. DNP is slow and higher throughput can be achieved by multi-sample polarization, but methods discussed in the previous section can accelerate the process. Sterility of the product is a strict requirement. Though the method involved is a simple dissolution it is nevertheless a compounding operation that requires several pharmaceuticals to be mixed at quite extreme conditions (e.g. $1 \mathrm{~K}$ for the solid sample and $400 \mathrm{~K}$ for the solvent). In some cases, the dissolution involves extreme $\mathrm{pH}$ and neutralization of the sample. The dose has to be sufficient for human subjects and product safety and efficacy needs to be assured through proper quality control or validation. Sterility of the sample needs to be maintained by a proper sterile barrier. The SPINlab (GE Healthcare, [49], fig 1) solves some of these problems. It operates at $5 \mathrm{~T}$ and $0.9 \mathrm{~K}$ in a closed cycle operation. There is no loss of cryogens in the operation. The cryostat consists of a superconducting magnet with liquid helium, but the cooling power is provided by a cryo cooler. The sample cooling is provided by a sorption pump, which is simply a canister filled with charcoal that will adsorb helium when cooled and releases the helium gas again when heated. The charcoal is cooled by closing a thermal switch connecting the sorption pump to the cryocooler driving the temperature of the charcoal to 5-6 K. The helium gas adsorbs, the pressure drops and the helium bath temperature drops. This can go on until the charcoal saturates and the helium bath runs dry. The adsorbed helium can then be regenerated by opening the thermal switch, heating the charcoal electrically, and condensing the released helium gas back into the helium vessel surrounding the sample space. The sample space can contain up to four samples simultaneously and has four independent loading systems. On the top side of the gate valves, are four air locks that together with the sliding seal on the fluid path allows loading and unloading of samples without breaking the vacuum of the 
[Type here]

sample space. This improves throughput and reduces dramatically heat load to helium bath. By gradually inserting the sample, more than $95 \%$ of the sample heat is rejected to the cryocooler and only $30 \mathrm{~J}$ ends up in the bath for a $1 \mathrm{~g}$ sample. With this design a base temperature of less than $0.9 \mathrm{~K}$ can be sustained for more than three days. When the system is heavily used, the heat loads increases, but full day operation is achieved. The system is regenerated overnight to be ready for next day use.

The SPINlab uses a closed fluid path to provide the sterile barrier. It is a sealed plastic component with all the pharmaceuticals pre-loaded. It can be single use and disposable for clinical use, but can be reused for non-sterile applications (in vitro and animal research). It has a vial that can hold up to $2 \mathrm{~mL}$ of sample. The vial is glued or welded to a long tube that allows it to reach the low temperature region. The other end of the tube is connected to a syringe with the solvent that sits outside the cryostat in a heater. Inside this outer tube is an inner tube, which allows solvent to be ejected onto the sample during dissolution. The syringe allows the solvent to be heated to a desired temperature and released at a controlled flow rate. The dissolved sample returns along the outer tubing, past the syringe, and into the receiver where all the quality control is performed. A sliding seal on the outer tubing allows the sample to be moved through the cryostat without breaking the vacuum. In contrast to other published dissolution systems, where the solvent vapor pressure is the drive pressure for the solvent, this design allows independent control of solvent temperature and flow rate, which is critical in ensuring an optimal dissolution and full preservation of polarization for more complicated samples [50]. The SPINlab fluid path also has the advantage that the all-plastic component does not leach any paramagnetic metals into the sample that can reduce $T_{1}$ of the hyperpolarized agent. The dissolution aspect has in general not been well characterized, and optimizing the fluid dynamics to ensure a fast 
[Type here]

and complete dissolution in a controlled manor is essential to minimize relaxation. The optimization involves understanding the heat transfer from the solvent to the fluid path, the flow pattern in the sample vial and heat transfer at the liquid-solid interface.

Quality control is essential for clinical use of hyperpolarization. Dissolution-DNP does not involve any chemistry, but is a complex compounding process. In the case of pyruvate, the most used formulation is based on pyruvic acid with trityl, and it involves a neutralization step. The pyruvic acid formulation is advantageous since neat pyruvic acid is $14 \mathrm{M}$, and a human dose can be achieved with ca $1.5 \mathrm{~g}$ of pyruvic acid (or $1.2 \mathrm{~mL}$ ). Other formulations with lower molarity require correspondingly larger sample volume. This is a requirement that needs to be considered for any agent formulation that will be translated to the clinic. In the case of pyruvate, the quality control involves measurement of $\mathrm{pH}$, temperature, volume, pyruvate concentration, radical concentration (to verify removal) and polarization. These measurements are performed optically and by NMR without product contact. The time from start of dissolution to completion of all QC measurements is ca $30 \mathrm{~s}$ for the SPINlab. This is impressive, but also illustrates that hyperpolarization for clinical use is a balance between process validation and QC. Even with $30 \mathrm{~s}$, plus the time to injection, significant polarization is lost for agents with relatively short $T_{1}$ (e.g. glucose with a $T_{1}$ of ca 15 s). Hyperpolarization in general, pushes the boundaries of "bed side" pharmacy and safety and efficacy needs to be demonstrated by validation or quality control. The position of regulators will be important, and will have significant influence on the medical application of hyperpolarization.

For hyperpolarization to have a role in diagnostic imaging relies on finding indications with a real unmet medical need. The first clinical study with a hyperpolarized injectable was in male patients with confirmed prostate cancer [51]. The study was a 
[Type here]

feasibility study, and the aims were to optimize the imaging protocol, demonstrate that cancer could be detected from the lactate image and safety. The dynamic spectroscopy showed rapid bolus arrival of the hyperpolarized $\left[1-{ }^{13} \mathrm{C}\right]$ pyruvate to the prostate, within 30 s; no detectable lactate in the normal prostate, but a large lactate metabolite signal in the slice containing the cancer. When knowing the pharmacokinetics, the imaging could be initiated at the optimal time point, to generate metabolic maps from an EPSI sequence. Fig 7 shows an example of a patient that had a known abnormality in the right gland on the $T_{2}$ proton image, but nothing noticeable on the left side. However, the lactate/pyruvate image showed bilateral disease, which was later confirmed by pathology. The above study was performed with prototype polarizer similar to the one described in fig $1 \mathrm{~A}$ installed in a clean room. Recently, Dr Keshari, MSKCC, reported that the first patients have been imaged with hyperpolarized pyruvate using the SPINlab. With currently about fifteen SPINlabs installed worldwide, the coming years opens up for multi-site clinical trials. For the field as a whole, it is essential that the clinical research generate important results.

\section{Concluding remarks}

Dissolution-DNP is a research field in the infancy. However, the science is flourishing. A perspectives article is likely to miss the target. Do we know the killer applications in chemistry, biology and medicine? Most likely not. More research is needed to understand for example, which unmet medical needs can be served, or the diversity of chemical reactions that can be followed. Do we understand the limitations of the technology? Most likely not. Even though a polarization close to unity can be reached by dissolution-DNP, the limits are constantly pushed. At this point in time, polarizers are expensive and complicated devices, available in few labs. To make hyperpolarization generally available requires not 
[Type here]

only engineering, but basic scientific breakthroughs. This work describes some of the current trends in field, as well as outlines some of the areas where new ideas will have an impact, with the hope that smarter people will make new discoveries.

\section{Acknowledgement}

The Danish National Research Foundation, grant number DNRF124, is gratefully acknowledged. 
[Type here]

\section{References}

[1] Y. Matsuki, S. Nakamura, S. Fukui, H. Suematsu, T. Fujiwara, Closed-cycle cold helium magic-angle spinning for sensitivity-enhanced multi-dimensional solid-state NMR., J. Magn. Reson. 259 (2015) 76-81. doi:10.1016/j.jmr.2015.08.003.

[2] E. Bouleau, P. Saint-Bonnet, F. Mentink-Vigier, H. Takahashi, J.-F. Jacquot, M. Bardet, et al., Pushing NMR sensitivity limits using dynamic nuclear polarization with closedloop cryogenic helium sample spinning, Chem. Sci. 6 (2015) 6806-6812. doi:10.1039/C5SC02819A.

[3] C.-G. Joo, K.-N. Hu, J. a Bryant, R.G. Griffin, In situ temperature jump high-frequency dynamic nuclear polarization experiments: enhanced sensitivity in liquid-state NMR spectroscopy., J. Am. Chem. Soc. 128 (2006) 9428-32. doi:10.1021/ja0611947.

[4] M. Sharma, G. Janssen, J. Leggett, A.P.M. Kentgens, P.J.M. van Bentum, Rapid-melt Dynamic Nuclear Polarization, J. Magn. Reson. 258 (2015) 40-48. doi:10.1016/j.jmr.2015.06.007.

[5] Device and method for producing a hyperpolarised nmr sample, EP1330182 B1, 2000. https://www.google.dk/patents/EP1330182B1?cl=en\&dq=ardenkjaer+melting\&hl=en \&sa=X\&ved=0ahUKEwiL2Y7m7IvKAhXFJw4KHQ2HChcQ6AEIUTAH (accessed January 2, 2016).

[6] R.W. Adams, J. a Aguilar, K.D. Atkinson, M.J. Cowley, P.I.P. Elliott, S.B. Duckett, et al., Reversible interactions with para-hydrogen enhance NMR sensitivity by polarization transfer., Science. 323 (2009) 1708-11. doi:10.1126/science.1168877.

[7] M.S. Albert, G.D. Cates, B. Driehuys, W. Happer, B. Saam, C.S. Springer, et al., Biological magnetic resonance imaging using laser-polarized 129Xe, Nature. 370 (1994) 199-201. doi:10.1038/370199a0.

[8] C.R. Bowers, D.P. Weitekamp, Transformation of Symmetrization Order to NuclearSpin Magnetization by Chemical Reaction and Nuclear Magnetic Resonance, Phys. Rev. Lett. 57 (1986) 2645-2648. doi:10.1103/PhysRevLett.57.2645.

[9] C.R. Bowers, D.P. Weitekamp, Parahydrogen and synthesis allow dramatically enhanced nuclear alignment, J. Am. Chem. Soc. 109 (1987) 5541-5542. doi:10.1021/ja00252a049.

[10] K. Golman, O. Axelsson, H. Jóhannesson, S. Månsson, C. Olofsson, J.S. Petersson, Parahydrogen-induced polarization in imaging: subsecond (13)C angiography., Magn. Reson. Med. 46 (2001) 1-5. http://www.ncbi.nlm.nih.gov/pubmed/11443703.

[11] J.R. Owers-Bradley, A.J. Horsewill, D.T. Peat, K.S.K. Goh, D.G. Gadian, High polarization of nuclear spins mediated by nanoparticles at millikelvin temperatures., Phys. Chem. Chem. Phys. 15 (2013) 10413-7. doi:10.1039/c3cp51274f.

[12] M.L. Hirsch, N. Kalechofsky, A. Belzer, M. Rosay, J.G. Kempf, Brute-Force Hyperpolarization for NMR and MRI., J. Am. Chem. Soc. 137 (2015) 8428-34. doi:10.1021/jacs.5b01252.

[13] M.C. Krishna, S. English, K. Yamada, J. Yoo, R. Murugesan, N. Devasahayam, et al., 
Overhauser enhanced magnetic resonance imaging for tumor oximetry: coregistration of tumor anatomy and tissue oxygen concentration., Proc. Natl. Acad. Sci. U. S. A. 99 (2002) 2216-21. doi:10.1073/pnas.042671399.

[14] J.H. Ardenkjær-Larsen, I. Laursen, I. Leunbach, G. Ehnholm, L.-G.G. Wistrand, J.S.S. Petersson, et al., EPR and DNP Properties of Certain Novel Single Electron Contrast Agents Intended for Oximetric Imaging, J. Magn. Reson. 133 (1998) 1-12. doi:10.1006/jmre.1998.1438.

[15] W. Boer, W. de Boer, Dynamic orientation of nuclei at low temperatures, J. Low Temp. Phys. 22 (1976) 185-212. doi:10.1007/BF00655221.

[16] J.H. Ardenkjaer-Larsen, B. Fridlund, A. Gram, G. Hansson, L. Hansson, M.H. Lerche, et al., Increase in signal-to-noise ratio of $>10,000$ times in liquid-state NMR., Proc. Natl. Acad. Sci. U. S. A. 100 (2003) 10158-10163. doi:10.1073/pnas.1733835100.

[17] H. Jóhannesson, S. Macholl, J.H. Ardenkjaer-Larsen, Dynamic Nuclear Polarization of [1-13C]pyruvic acid at 4.6 tesla., J. Magn. Reson. 197 (2009) 167-175. doi:10.1016/j.jmr.2008.12.016.

[18] W. Meyer, J. Heckmann, C. Hess, E. Radtke, G. Reicherz, L. Triebwasser, et al., Dynamic polarization of $13 \mathrm{C}$ nuclei in solid $13 \mathrm{C}$ labeled pyruvic acid, Nucl. Instruments Methods Phys. Res. Sect. A Accel. Spectrometers, Detect. Assoc. Equip. 631 (2011) 15. doi:10.1016/j.nima.2010.10.156.

[19] S. Jannin, a Comment, F. Kurdzesau, J. a Konter, P. Hautle, B. van den Brandt, et al., A $140 \mathrm{GHz}$ prepolarizer for dissolution dynamic nuclear polarization., J. Chem. Phys. 128 (2008) 241102. doi:10.1063/1.2951994.

[20] S. Jannin, A. Bornet, R. Melzi, G. Bodenhausen, High field dynamic nuclear polarization at 6.7T: Carbon-13 polarization above $70 \%$ within $20 \mathrm{~min}$, Chem. Phys. Lett. 549 (2012) 99-102. doi:10.1016/j.cplett.2012.08.017.

[21] L. Lumata, Z. Kovacs, A.D. Sherry, C. Malloy, S. Hill, J. van Tol, et al., Electron spin resonance studies of trityl OX063 at a concentration optimal for DNP, Phys. Chem. Chem. Phys. 15 (2013) 9800-9807. doi:10.1039/C3CP50186H.

[22] M. Batel, M. Krajewski, A. Däpp, A. Hunkeler, B.H. Meier, S. Kozerke, et al., Dissolution dynamic nuclear polarization efficiency enhanced by Hartmann-Hahn cross polarization, Chem. Phys. Lett. 554 (2012) 72-76. doi:10.1016/j.cplett.2012.10.018.

[23] A.P. Linde, P. Linde, A. Joaquin, Application of cross polarisation techniques to dynamic nuclear polarisation dissolution experiments, Nottingham University, 2010. http://etheses.nottingham.ac.uk/1417/ (accessed August 22, 2014).

[24] A. Bornet, R. Melzi, A.J. Perez Linde, P. Hautle, B. van den Brandt, S. Jannin, et al., Boosting Dissolution Dynamic Nuclear Polarization by Cross Polarization, J. Phys. Chem. Lett. 4 (2013) 111-114. doi:10.1021/jz301781t.

[25] A. Henstra, W.T. Wenckebach, Dynamic nuclear polarisation via the integrated solid effect I: theory, Mol. Phys. 0 (n.d.) 1-12. doi:10.1080/00268976.2013.861936.

[26] A. Henstra, W.T. Wenckebach, The theory of nuclear orientation via electron spin 
locking (NOVEL), Mol. Phys. (2008). http://www.tandfonline.com/doi/abs/10.1080/00268970801998262 (accessed October 20, 2015).

[27] J.H. Ardenkjaer-Larsen, S. Macholl, H. Jóhannesson, Dynamic Nuclear Polarization with Trityls at 1.2 K, Appl. Magn. Reson. 34 (2008) 509-522. doi:10.1007/s00723-0080134-4.

[28] E. Ravera, D. Shimon, A. Feintuch, D. Goldfarb, S. Vega, A. Flori, et al., The effect of Gd on trityl-based dynamic nuclear polarisation in solids., Phys. Chem. Chem. Phys. 17 (2015) 26969-78. doi:10.1039/c5cp04138d.

[29] S.C. Serra, M. Filibian, P. Carretta, A. Rosso, F. Tedoldi, Relevance of electron spin dissipative processes to dynamic nuclear polarization via thermal mixing, Phys. Chem. Chem. Phys. 16 (2013) 753-764. doi:10.1039/C3CP52534A.

[30] A. Comment, B. van den Brandt, K. Uffmann, F. Kurdzesau, S. Jannin, J.A. Konter, et al., Principles of Operation of a DNP Prepolarizer Coupled to a Rodent MRI Scanner, Appl. Magn. Reson. 34 (2008) 313-319. doi:10.1007/s00723-008-0119-3.

[31] M. Batel, M. Krajewski, K. Weiss, O. With, A. Däpp, A. Hunkeler, et al., A multi-sample $94 \mathrm{GHz}$ dissolution dynamic-nuclear-polarization system., J. Magn. Reson. 214 (2012) 166-74. doi:10.1016/j.jmr.2011.11.002.

[32] Y. Crémillieux, F. Goutailler, B. Montcel, D. Grand, G. Vermeulen, P.E. Wolf, A SuperWide Bore DNP System for Multiple Sample Polarization: Cryogenic Performance and Polarization at Low Temperature, Appl. Magn. Reson. 43 (2012) 167-180. doi:10.1007/s00723-012-0354-5.

[33] L.L. Lumata, R. Martin, A.K. Jindal, Z. Kovacs, M.S. Conradi, M.E. Merritt, Development and performance of a 129-GHz dynamic nuclear polarizer in an ultra-wide bore superconducting magnet., MAGMA. 28 (2015) 195-205. doi:10.1007/s10334-0140455-2.

[34] S. Bowen, J.H. Ardenkjaer-Larsen, Enhanced performance large volume dissolutionDNP, J. Magn. Reson. 240 (2014) 90-4. doi:10.1016/j.jmr.2014.01.009.

[35] H.-Y. Chen, C. Hilty, Implementation and Characterization of Flow Injection in Dissolution Dynamic Nuclear Polarization NMR Spectroscopy, ChemPhysChem. 16 (2015) 2646-2652. doi:10.1002/cphc.201500292.

[36] S. Bowen, C. Hilty, Rapid sample injection for hyperpolarized NMR spectroscopy., Phys. Chem. Chem. Phys. 12 (2010) 5766-70. doi:10.1039/c002316g.

[37] R. Sriram, M. Van Criekinge, A. Hansen, Z.J. Wang, D.B. Vigneron, D.M. Wilson, et al., Real-time measurement of hyperpolarized lactate production and efflux as a biomarker of tumor aggressiveness in an MR compatible 3D cell culture bioreactor., NMR Biomed. 28 (2015) 1141-9. doi:10.1002/nbm.3354.

[38] T. Cheng, M. Mishkovsky, J.A.M. Bastiaansen, O. Ouari, P. Hautle, P. Tordo, et al., Automated transfer and injection of hyperpolarized molecules with polarization measurement prior to in vivo NMR., NMR Biomed. 26 (2013) 1582-8. doi:10.1002/nbm.2993. 
[Type here]

[39] P. Miéville, P. Ahuja, R. Sarkar, S. Jannin, P.R. Vasos, S. Gerber-Lemaire, et al., Scavenging free radicals to preserve enhancement and extend relaxation times in NMR using dynamic nuclear polarization., Angew. Chem. Int. Ed. Engl. 49 (2010) 6182-5. doi:10.1002/anie.201000934.

[40] J. Milani, B. Vuichoud, A. Bornet, P. Miéville, R. Mottier, S. Jannin, et al., A magnetic tunnel to shelter hyperpolarized fluids., Rev. Sci. Instrum. 86 (2015) 024101. doi:10.1063/1.4908196.

[41] E. Chiavazza, E. Kubala, C. V Gringeri, S. Düwel, M. Durst, R.F. Schulte, et al., Earth's magnetic field enabled scalar coupling relaxation of $13 \mathrm{C}$ nuclei bound to fast-relaxing quadrupolar 14N in amide groups., J. Magn. Reson. 227 (2013) 35-8. doi:10.1016/j.jmr.2012.11.016.

[42] G.D. Reed, C. von Morze, R. Bok, B.L. Koelsch, M. Van Criekinge, K.J. Smith, et al., High resolution (13)C MRI with hyperpolarized urea: in vivo T(2) mapping and (15)N labeling effects., IEEE Trans. Med. Imaging. 33 (2014) 362-71. doi:10.1109/TMI.2013.2285120.

[43] G. Pileio, S. Bowen, C. Laustsen, M.C.D. Tayler, J.T. Hill-Cousins, L.J. Brown, et al., Recycling and imaging of nuclear singlet hyperpolarization, Am. Chem. Soc. J. 135 (2013) 5084-5088. doi:10.1021/ja312333v.

[44] T. Harris, C. Bretschneider, L. Frydman, Dissolution DNP NMR with solvent mixtures: Substrate concentration and radical extraction, J. Magn. Reson. 211 (2011) 96-100. doi:10.1016/j.jmr.2011.04.001.

[45] T. Harris, O. Szekely, L. Frydman, On the Potential of Hyperpolarized Water in Biomolecular NMR Studies, J. Phys. Chem. B. 118 (2014) 3281-3290. doi:10.1021/jp4102916.

[46] A. Capozzi, J.-N. Hyacinthe, T. Cheng, T.R. Eichhorn, G. Boero, C. Roussel, et al., Photoinduced Nonpersistent Radicals as Polarizing Agents for X-Nuclei Dissolution Dynamic Nuclear Polarization, J. Phys. Chem. C. 119 (2015) 22632-22639. doi:10.1021/acs.jpcc.5b07315.

[47] D. Gajan, A. Bornet, B. Vuichoud, J. Milani, R. Melzi, H.A. van Kalkeren, et al., Hybrid polarizing solids for pure hyperpolarized liquids through dissolution dynamic nuclear polarization., Proc. Natl. Acad. Sci. U. S. A. 111 (2014) 14693-7. doi:10.1073/pnas.1407730111.

[48] S. Jannin, No Title, in: 5th Int. Hyperpolarized Magn. Reson. Meet., Egmond ann Zee, NL, 2015.

[49] J.H. Ardenkjaer-Larsen, A.M. Leach, N. Clarke, J. Urbahn, D. Anderson, T.W. Skloss, Dynamic nuclear polarization polarizer for sterile use intent., NMR Biomed. 24 (2011) 927-932. doi:10.1002/nbm.1682.

[50] J. Jam, S. Dey, L. Muralidharan, A.M.A.M. Leach, J.H. Ardenkjaer-Larsen, Jet impingment melting with vaporization: A numerical study, in: 2008 Proc. ASME Summer Heat Transf. Conf. HT 2008, 2009: pp. 559-567. http://www.scopus.com/inward/record.url?eid=2-s2.070349120429\&partnerID=tZOtx3y1. 
[Type here]

[51] S.J. Nelson, J. Kurhanewicz, D.B. Vigneron, P.E.Z. Larson, A.L. Harzstark, M. Ferrone, et al., Metabolic imaging of patients with prostate cancer using hyperpolarized [1${ }^{13}$ C]pyruvate., Sci. Transl. Med. 5 (2013) 198ra108.

doi:10.1126/scitranslmed.3006070. 
[Type here]

\section{Figure legends}

Fig 1: Polarizer generations: A) Original PNAS 2003 design: Schematic drawing of the DNP polarizer and parts. 1, DNP polarizer; 2, vacuum pump; 3, VTI; 4, microwave source; 5, pressure transducer; 6 , sample port; 7 , microwave container; 8 , sample holder; 9 , sample container; 10, dissolution wand, B) Hypersense, Oxford Instruments PIc and C) SPINlab, GE Healthcare. The Hypersense is based on the design of the original 2003 polarizer and operates at $3.35 \mathrm{~T}$ and $1.4 \mathrm{~K}$. The SPINlab operates at $5 \mathrm{~T}$ and $0.9 \mathrm{~K}$. Figure $1 \mathrm{~A}$ is reprinted with permission from J.H. Ardenkjaer-Larsen, B. Fridlund, A. Gram, G. Hansson, L. Hansson, M.H. Lerche, et al., Increase in signal-to-noise ratio of $>10,000$ times in liquid-state NMR., Proc. Natl. Acad. Sci. U. S. A. 100 (2003) 10158-10163. Copyright PNAS.

Fig 2: A. Maximum ${ }^{13} \mathrm{C}$ polarization of $\left[1-{ }^{13} \mathrm{C}\right]$ pyruvic acid (sample $\mathrm{A}$ is $10 \mathrm{mM}$ and sample $\mathrm{B}$ is $15 \mathrm{mM}$ trityl (AH111501)) at different magnetic field strengths from $2.5 \mathrm{~T}$ to $5 \mathrm{~T}$. B. Maximum ${ }^{13} \mathrm{C}$ polarization at $5 \mathrm{~T}$ for sample $\mathrm{B}$ for different temperatures from $0.9 \mathrm{~K}$ to 1.35 K. Reprinted with permission from W. Meyer, J. Heckmann, C. Hess, E. Radtke, G. Reicherz, L. Triebwasser, et al., Dynamic polarization of $13 \mathrm{C}$ nuclei in solid $13 \mathrm{C}$ labeled pyruvic acid, Nucl. Instruments Methods Phys. Res. Sect. A Accel. Spectrometers, Detect. Assoc. Equip. 631 (2011) 1-5. Copyright 2010 Elsevier B.V.

Fig 3: Cross-polarization (CP) sequence in dissolution-DNP. The sample is placed in a polarizer at $1.2 \mathrm{~K}$ and $6.7 \mathrm{~T}$ and subjected to continuous microwave irradiation at $188.3 \mathrm{GHz}$ and $120 \mathrm{~mW}$. (a) A train of $30 \pi / 2$ pulses is applied to both ${ }^{1} \mathrm{H}$ and ${ }^{13} \mathrm{C}$ for presaturation. (b) Adiabatic half-passage pulses ( $250 \mathrm{us}, 15 \mathrm{kHz}$ ) to convert the magnetization from 
[Type here]

longitudinal to transverse followed by adiabatic passage through the Hartmann-Hahn condition (optional). (c) A sequence of 8 pulses with $5^{\circ}$ degree flip angle separated by $30 \mathrm{~s}$ is applied to monitor the buildup of the polarization. This scheme is repeated every $240 \mathrm{~s}$ until the desired level of ${ }^{13} \mathrm{C}$ polarization is achieved. (A) Signals of ${ }^{13} \mathrm{C} N M R$ of $50 \mu \mathrm{L}$ of a $3 \mathrm{M}$ frozen solution of $\left[1-{ }^{13} \mathrm{C}\right]$ acetate in a deuterated water/ethanol mixture $(67: 33 \mathrm{v} / \mathrm{v})$ doped with $50 \mathrm{mM}$ TEMPO measured without $\mathrm{CP}$ (blue) at thermal equilibrium ( $\mathrm{T}=4.2 \mathrm{~K}$ and $\mathrm{B}_{0}=$ 6.7 T, scaled by a factor 100) and with CP-DNP (red) (C) ${ }^{13} \mathrm{C}$ DNP buildup with (red) and without (gray) (C) The dissolution and transfer process takes $10.7 \mathrm{~s}$. (D) Relaxation of ${ }^{13} \mathrm{C}$ in solution at $300 \mathrm{~K}$ after transfer to a magnetic field $\mathrm{B}_{0}=7.05 \mathrm{~T}$, measured with $\beta=5^{\circ}$ pulses at 5 s intervals. (E) Signals of $\left[1-{ }^{13} \mathrm{C}\right]$ acetate immediately after dissolution (red) and at thermal equilibrium (blue, scaled by a factor 2048). Adapted with permission from A. Bornet, R. Melzi, A.J. Perez Linde, P. Hautle, B. van den Brandt, S. Jannin, et al., Boosting Dissolution Dynamic Nuclear Polarization by Cross Polarization, J. Phys. Chem. Lett. 4 (2013) 111-114. Copyright 2012 American Chemical Society.

FIG 4: a) Schematic of the liquid-driven injector. Hyperpolarized samples and nonhyperpolarized reactants can be loaded into two sample loops. b) The status of the syringe pumps and two-position valves (V1 and V2) during the experiment. A solid bar indicates that the valve is in the loading position (L), and a hollow bar indicates the injection position (I). C) The sample transfer is stabilized in less than a second. Adapted with permission from H.-Y. Chen, C. Hilty, Implementation and Characterization of Flow Injection in Dissolution Dynamic Nuclear Polarization NMR Spectroscopy, ChemPhysChem. 16 (2015) 2646-2652, copyright 2015 Wiley, and S. Bowen, C. Hilty, Rapid sample injection for hyperpolarized 
[Type here]

NMR spectroscopy, Phys. Chem. Chem. Phys. 12 (2010) 5766-70, copyright 2010 the Owner Societies.

Fig 5: Radical concentration generated by UV irradiation at $77 \mathrm{~K}$ as a function of the illumination time for frozen beads $(8.0 \pm 0.5 \mu \mathrm{L})$ of neat pyruvic acid (black diamonds), pyruvic acid:ethanol 1:1 (v/v) (red circles), and pyruvic acid:THF 1:1 (v/v). ${ }^{13} \mathrm{C}$ and ${ }^{1} \mathrm{H}$ DNP microwave spectra measured at $5 \mathrm{~T}$ and $1.5 \mathrm{~K}$ of pyruvic acid and pyruvic acid- $\mathrm{d}_{4}$ after 60 min of UV irradiation. The simulated ESR spectrum for each of the radicals is superimposed (solid gray lines). Adapted with permission from A. Capozzi, J.-N. Hyacinthe, T. Cheng, T.R. Eichhorn, G. Boero, C. Roussel, et al., Photoinduced Nonpersistent Radicals as Polarizing Agents for X-Nuclei Dissolution Dynamic Nuclear Polarization, J. Phys. Chem. C. 119 (2015) 22632-22639. Copyright 2015 American Chemical Society.

Fig 6: Hyperpolarization by dissolution-DNP with HYPSO. (A) HYPSO 1.0 is impregnated with a solution of the analyte to be polarized without addition of any glass forming agents. The transmission electron microscope image shows the porous structure of the material. The red dots schematically represent the polarizing agent. (B) Proton DNP is performed on 20 mg HYPSO 1.0 material $(88 \mu \mathrm{mol} / \mathrm{g})$ impregnated with $36 \mu \mathrm{L} 3 \mathrm{M}$ solution of $\left[1-{ }^{13} \mathrm{C}\right]$ pyruvate in $\mathrm{D}_{2} \mathrm{O}$. The proton polarization rapidly builds up with a time constant of $119 \mathrm{~s}$, and by applying ${ }^{1} \mathrm{H}$ to ${ }^{13} \mathrm{C}$ cross polarization, a ${ }^{13} \mathrm{C}$ polarization of $20 \%$ is reached in $17 \mathrm{~min}$. (C) The DNP sample is dissolved and expelled from HYPSO 1.0 by injecting $5 \mathrm{~mL}$ superheated $\mathrm{D}_{2} \mathrm{O}$; a series of ${ }^{13} \mathrm{C}$ NMR spectra of $\left[1-{ }^{13} \mathrm{C}\right]$ pyruvate is measured every $5 \mathrm{~s}$. The liquid-state ${ }^{13} \mathrm{C}$ polarization was $25.3 \%$ with a $T_{1}$ of $49 \mathrm{~s}$. Reprinted with permission from D. Gajan, A. Bornet, B. Vuichoud, J. Milani, R. Melzi, H.A. van Kalkeren, et al., Hybrid polarizing solids for 
[Type here]

pure hyperpolarized liquids through dissolution dynamic nuclear polarization., Proc. Natl. Acad. Sci. U. S. A. 111 (2014) 14693-7. Copyright PNAS.

FIG 7: 2D single-time point MRSI data from a patient with serum PSA of $9.5 \mathrm{ng} / \mathrm{mL}$, who was diagnosed with bilateral biopsy-proven Gleason grade $3+3$ prostate cancer. The axial $T_{2}-$ weighted image shows a unilateral region of reduced signal intensity (red arrows), which is consistent with a reduction in the corresponding ADC. The ${ }^{1} \mathrm{H}$ MRS supported these findings, with voxels with reduced citrate and elevated choline/citrate (highlighted in pink) on the right side of the gland and voxels with normal metabolite ratios on the left side. The ${ }^{13} \mathrm{C}$ MRSI (0.43 mL/kg [1-13 C]pyruvate) show voxels with elevated levels of hyperpolarized lactate/pyruvate (highlighted in pink) on both the right and left sides of the prostate. Reprinted with permission from S.J. Nelson, J. Kurhanewicz, D.B. Vigneron, P.E.Z. Larson, A.L. Harzstark, M. Ferrone, et al., Metabolic imaging of patients with prostate cancer using hyperpolarized $\left[1{ }^{13}\right.$ C]pyruvate., Sci. Transl. Med. 5 (2013) 198ra108. Copyright 2013, The American Association for the Advancement of Science. 
[Type here]

FIG 1:

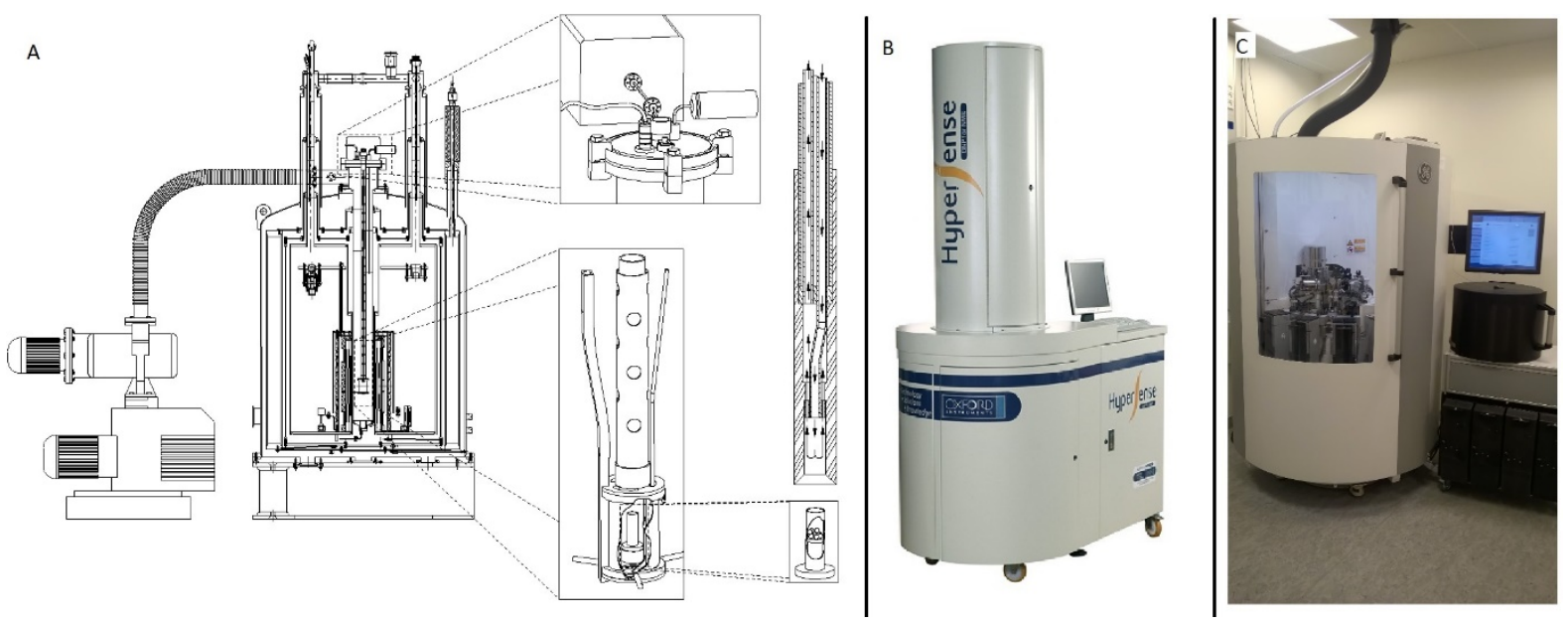


[Type here]

FIG 2:
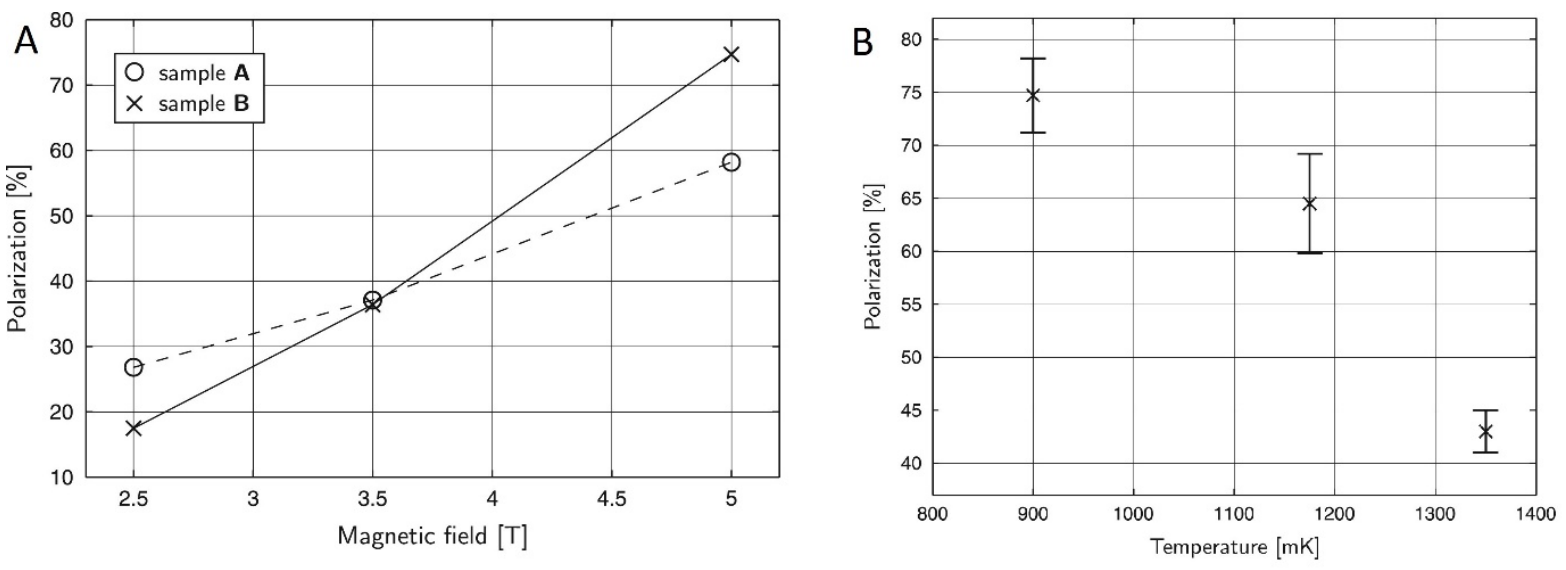
[Type here]

FIG 3:

(a)

(b)

(c)

(d)

(e)

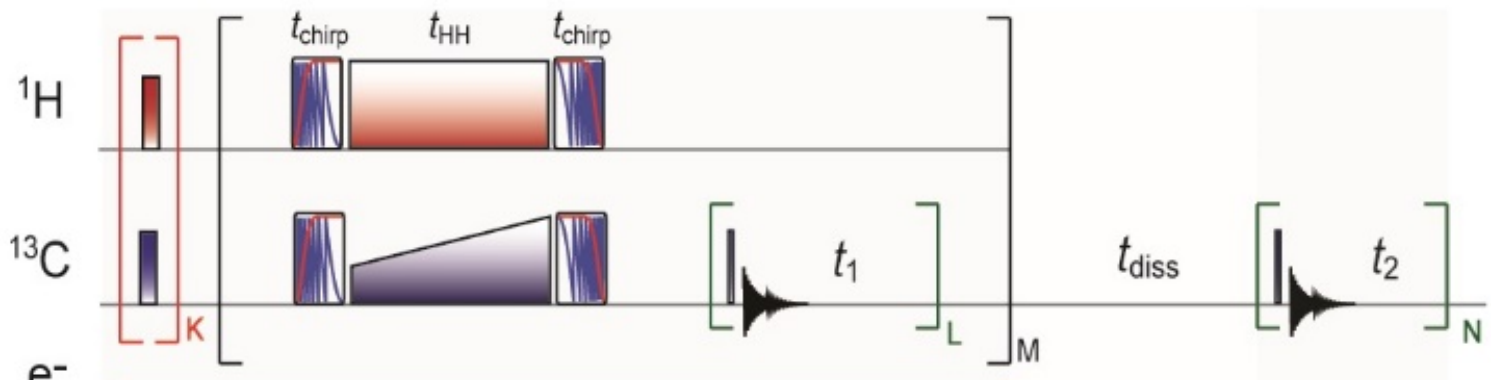

$\mathrm{e}^{-}$

$\mathrm{B}_{0}$

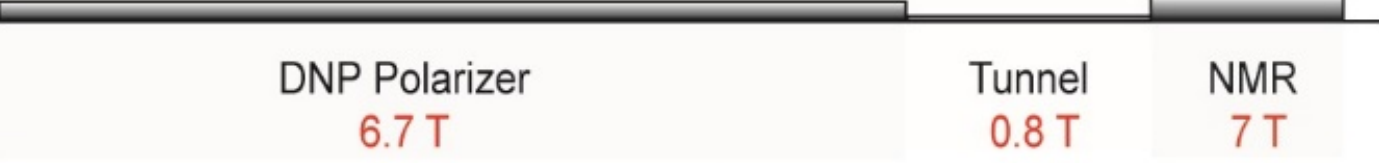

(A)
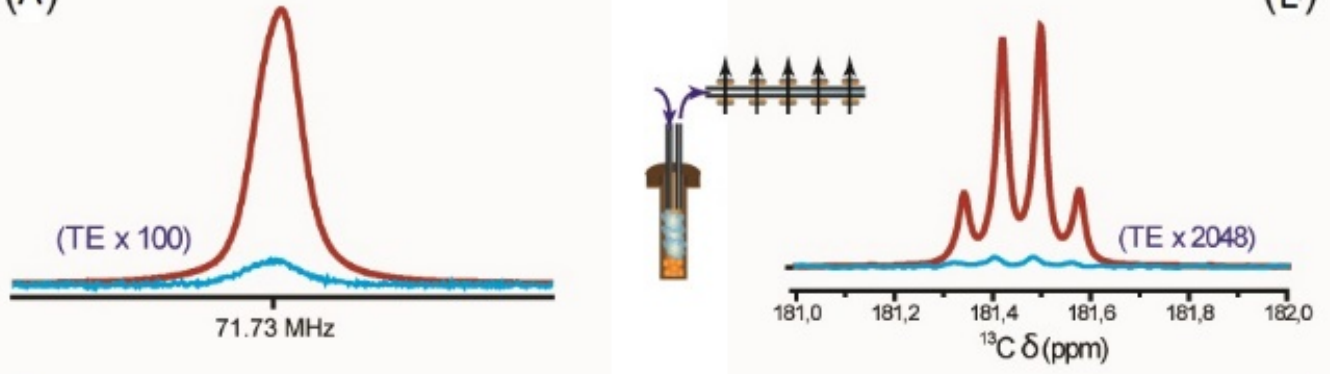

(B)

(c)

(D)
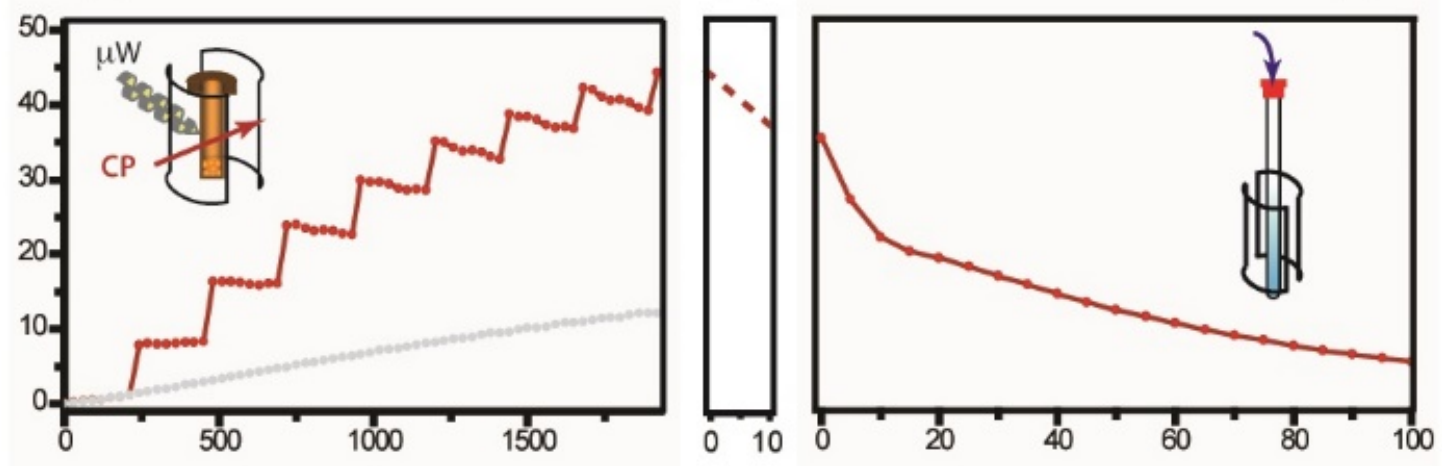

Time (s) 
[Type here]

FIG 7:
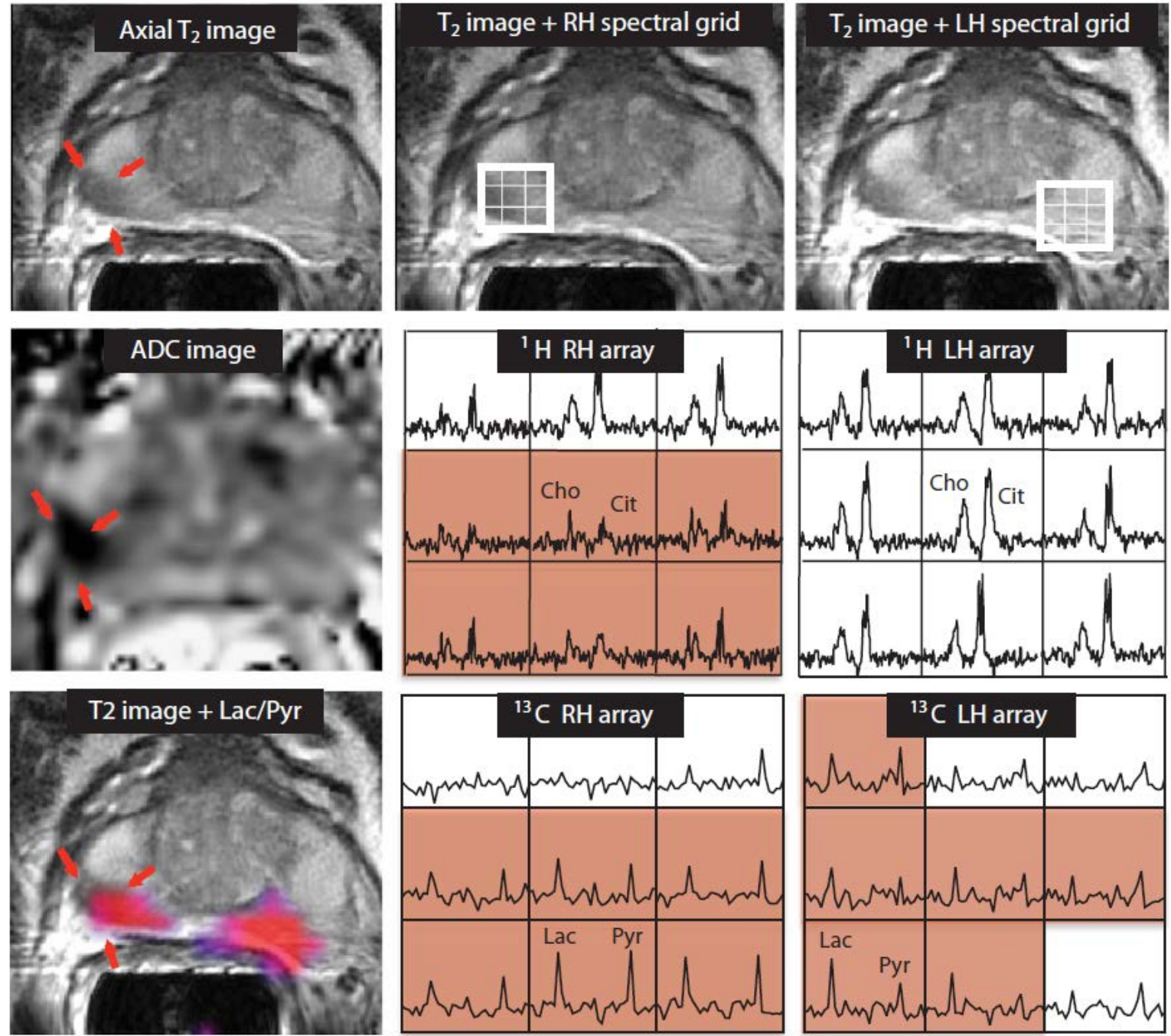

${ }^{13}$ C LH array


[Type here]

FIG 4:

(a)
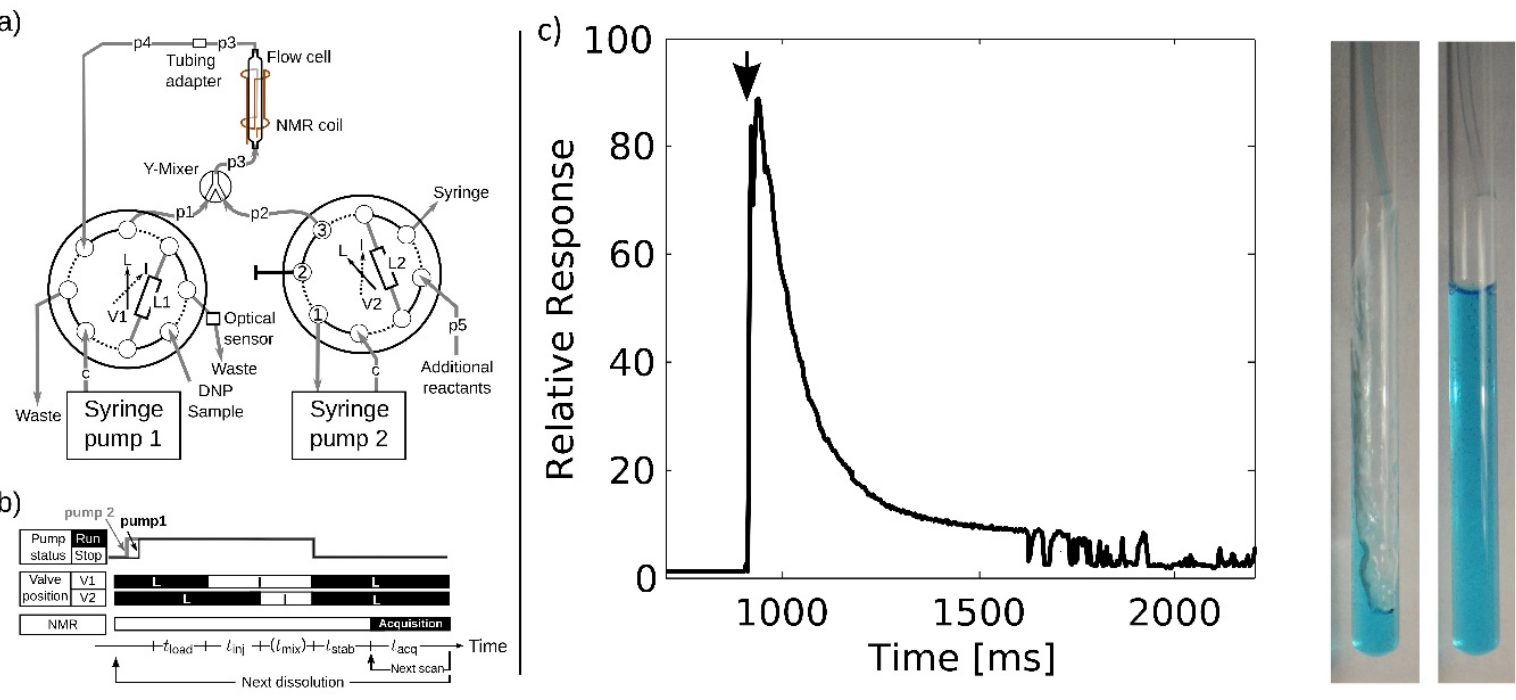
[Type here]

FIG 5:
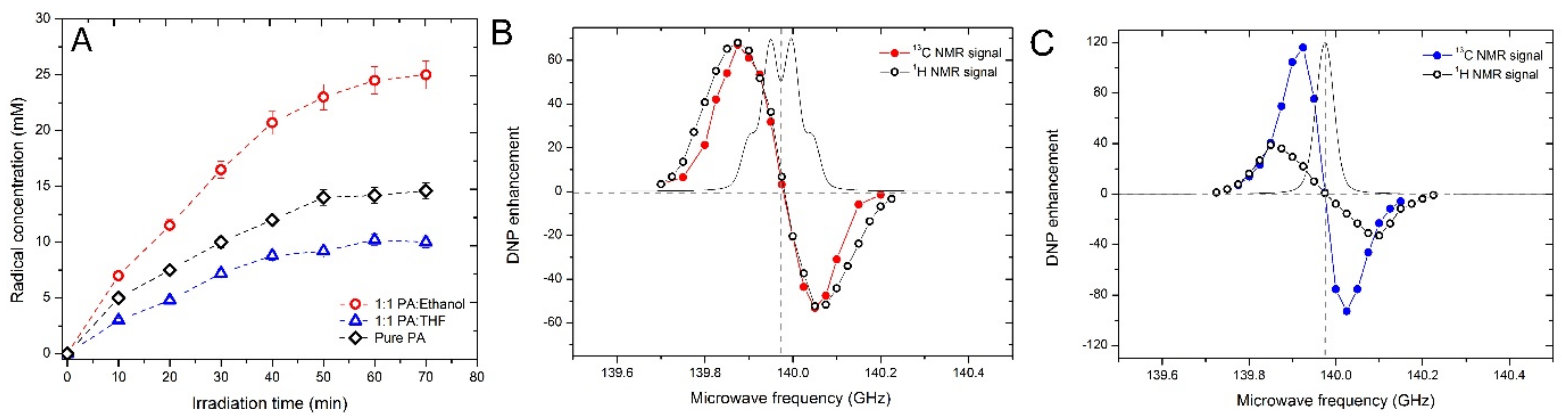
[Type here]

FIG 6:

a. HYPSO-1.0 impregnated with a $\left[1{ }^{13} \mathrm{C}\right]$ pyruvate solution.

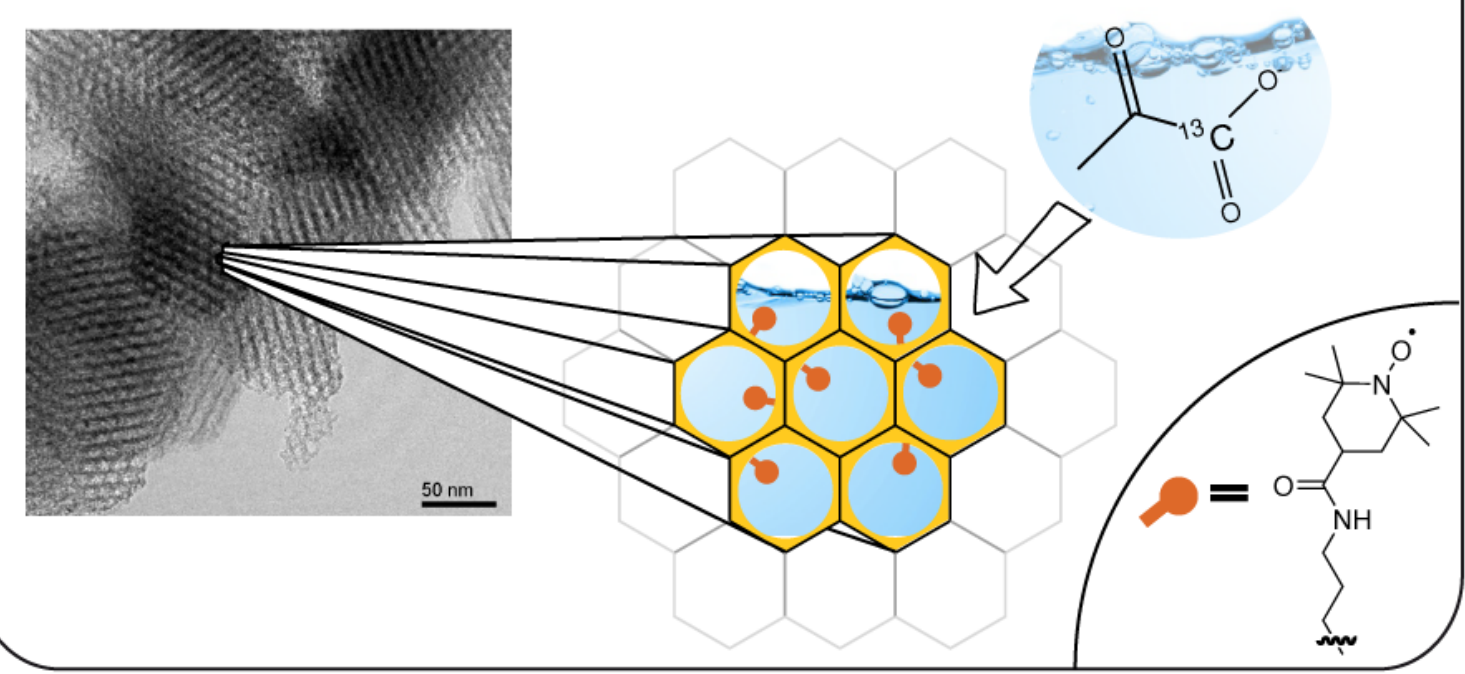

b. Low temperature $\mathrm{e}^{-} \rightarrow{ }^{1} \mathrm{H} \rightarrow{ }^{13} \mathrm{C}$ cross polarization DNP at $T=1.2 \mathrm{~K}$ and $B_{0}=6.7 \mathrm{~T}$.

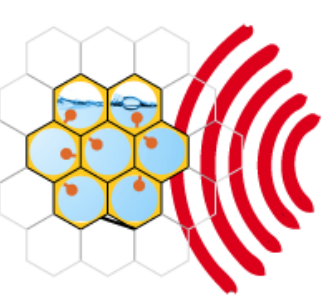

CW microwave irradiation and ${ }^{1} \mathrm{H} \rightarrow{ }^{13} \mathrm{C}$ CP transfer

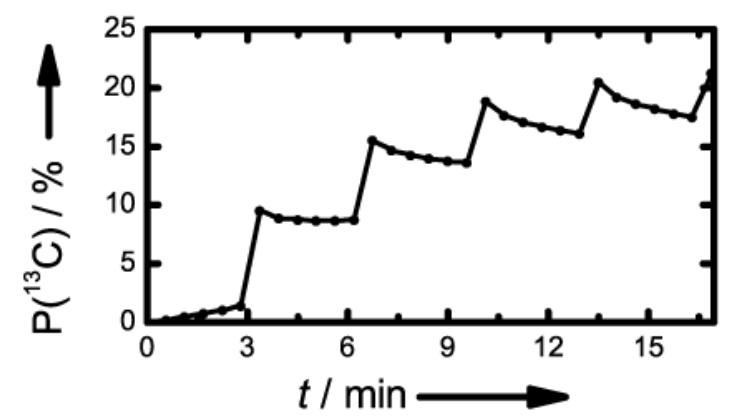

c. Hyperpolarization after dissolution.
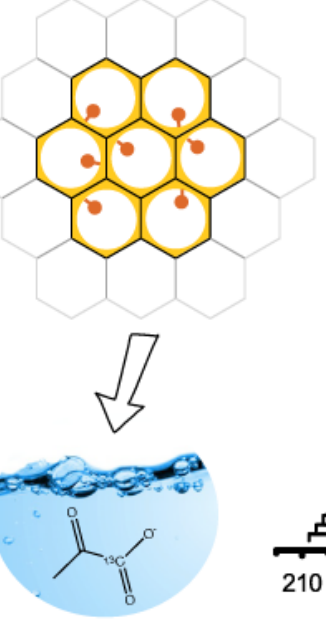

$$
\varepsilon_{D N P}>32^{\prime} 000
$$

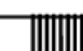

